\title{
Two- and three-pion interferometry for a nonchaotic source in relativistic nuclear collisions
}

\author{
Hiroki Nakamura ${ }^{1}$ and Ryoichi Seki ${ }^{2,3}$ \\ ${ }^{1}$ Department of Physics, Waseda University, Tokyo 169-8555, Japan \\ ${ }^{2}$ Department of Physics, California State University, Northridge, California 91330 \\ ${ }^{3}$ W. K. Kellogg Radiation Laboratory, 106-38, California Institute of Technology, Pasadena, California 91125
}

(Received 25 August 1999; published 14 April 2000)

\begin{abstract}
Two- and three-pion correlation functions are investigated for a source that is not fully chaotic. Various models are examined to describe the source. The chaoticity and weight factor are evaluated in each model as measures of the strength of correlations and compared to experimental results. A new measure of three-pion correlation is also suggested.
\end{abstract}

PACS number(s): $25.75 . G z$

\section{INTRODUCTION}

Two-pion correlations obtained in relativistic heavy-ion collisions have been used to extract the size and shape of the pion-emitting source, based on the Hanbury-Brown-Twiss (HBT) effect. Correlations are not, of course, limited to those of two pions, but can also be of multipions. Though multipion correlations are unavoidably complicated, they contain new information not available from the two-pion correlations. For example, when final-state interactions of the emitted pions are neglected, the two-pion correlations for a chaotic source depend only on the magnitude of the Fourier transform of the pion-source function, but the multipion correlations depend also on its phase $[1,2]$. The magnitude is an even function of the relative momentum of the emitted pions, while the phase is an odd function. One thus hopes to extract new information about the source from the multipion correlations.

In a previous paper [2], we made a detailed investigation of three-pion correlations, the simplest multipion correlations after the two-pion correlations, over a wide range of kinematics, to investigate the feasibility of extracting new information about the source through the phase in the case of a chaotic source. We find that extracting new information would be rather difficult in practice because the multiplicative factor of the phase function becomes small in the region where the phase varies appreciably.

In experiments, the two-pion correlations at the zero relative momentum is observed to be less than two. After the final-state interactions are removed, it must be two in the case of a chaotic source. The measurement suggests that the source is not completely chaotic. Recently, a measurement of the three-pion correlations has been reported, showing that the strength of the three-pion correlations are also less than expected in the case of a chaotic source.

In this paper we investigate the two- and three-pion correlations for a source not completely chaotic. We introduce models of the source that are various mixtures of coherent and chaotic sources. The models also include one with a novel structure, a mixture of multiple coherent sources and a chaotic source. These models describe different dynamics generating pion emissions, though we do not pursue the identification of dynamics corresponding to each model.

A mixture of a coherent source and a chaotic source is a model that has often appeared in the literature $[3,4,1]$ under the name "partially coherent source." We find that this popular model poorly reproduces the recent data of the twoand three-pion correlations [5]. A model consisting of multicoherent sources and one chaotic source appears to yield a good agreement.

It is both theoretical and experimental practice to calculate the so-called weight factor from the three-pion correlations at the vanishing relative momenta. The weight factor is usually considered to represent the strength of the genuine three-pion correlations. When we examine more complicated models than the popular partially coherent model, we find that the weight factor no longer describes the genuine strength. The expression that yields the genuine strength depends on the structure of the source, and there is no universal expression as such. Nevertheless, we propose a modified expression for the weight factor that has a wider validity.

In Sec. II, we define pion spectra, correlation functions, and measures of two- and three-pion correlation functions, chaoticity, and weight factor, respectively. In Sec. III, various nonchaotic models of the pion-emitting source are introduced, and chaoticities and weight factors are obtained for them. Section IV presents discussions and a summary: a new expression of the weight factor is introduced and discussed, and chaoticities and weight factors are compared with the recent experimental data. Appendix A gives a derivation of correlations for multicoherent sources. In Appendix B, the new weight factor is derived.

\section{CORRELATION FUNCTIONS, CHAOTICITY, AND WEIGHT FACTOR}

For the sake of clarity in the sections to follow, we define correlation functions and their measures, chaoticity and weight factor. Note that our definition is standard.

We first write the basic quantities, pion spectra, in second-quantization form, as follows:

$$
\begin{gathered}
W_{1}(p)=\left\langle a_{p}^{\dagger} a_{p}\right\rangle, \\
W_{2}\left(p_{1}, p_{2}\right)=\left\langle a_{p_{1}}^{\dagger} a_{p_{2}}^{\dagger} a_{p_{1}} a_{p_{2}}\right\rangle, \\
W_{3}\left(p_{1}, p_{2}, p_{3}\right)=\left\langle a_{p_{1}}^{\dagger} a_{p_{2}}^{\dagger} a_{p_{3}}^{\dagger} a_{p_{1}} a_{p_{2}} a_{p_{3}}\right\rangle .
\end{gathered}
$$


Though these expressions are simple and reasonable, the explicit definition of $\langle\ldots\rangle$ is a complicated issue. $\langle\ldots\rangle$ represents a quantum statistical average and is formally written as $\langle\psi|\ldots| \psi\rangle$ in terms of the quantum state, $|\psi\rangle$, or $\operatorname{Tr}\{\hat{\rho} \ldots\}$ in terms of the the density matrix, $\hat{\rho}$. In this work, we introduce models to represent various underlying dynamics of the pion emission. Note that the momenta above and hereafter are on-shell, e.g., $p^{0}=\sqrt{\mathbf{p}^{2}+m^{2}}$ for $p$.

In terms of the spectra, we define two- and three-pion correlation functions in the usual way [3],

$$
\begin{gathered}
C_{2}\left(p_{1}, p_{2}\right)=\frac{\langle n\rangle^{2}}{\langle n(n-1)\rangle} \frac{W_{2}\left(p_{1}, p_{2}\right)}{W_{1}\left(p_{1}\right) W_{1}\left(p_{2}\right)}, \\
C_{3}\left(p_{1}, p_{2}, p_{3}\right)=\frac{\langle n\rangle^{3}}{\langle n(n-1)(n-2)\rangle} \frac{W_{3}\left(p_{1}, p_{2}, p_{3}\right)}{W_{1}\left(p_{1}\right) W_{1}\left(p_{2}\right) W_{1}\left(p_{3}\right)} .
\end{gathered}
$$

Here, the normalizations are introduced in order to take account of multiplicity fluctuation, with the following definitions:

$$
\begin{gathered}
\langle n\rangle=\int d^{3} p W_{1}(p), \\
\langle n(n-1)\rangle=\int d^{3} p_{1} d^{3} p_{2} W_{2}\left(p_{1}, p_{2}\right),
\end{gathered}
$$

$$
\langle n(n-1)(n-2)\rangle=\int d^{3} p_{1} d^{3} p_{2} d^{3} p_{3} W_{2}\left(p_{1}, p_{2}, p_{3}\right) .
$$

In the usual models, the above normalizations yield $C_{2}$ and $C_{3} \rightarrow 1$ as the relative momenta approach infinity. In more complicated models, such as those that possess the particlenumber fluctuation per mode, however, the normalizations of Eqs. (4) and (5) yield an asymptotic value different from unity. We will discuss this point fully in Sec. III D.

When the HBT effect does not appear, correlation functions are independent of relative momenta. When the HBT effect occurs, however, the correlation functions are no longer constant, but are dependent on relative momenta. As is well known, the size and shape of a pion-emitting source are extracted from the relative-momentum dependence of the correlations through the HBT effect. The methods of extracting the size and shape of the source has been discussed numerous times, and we will not go into the issues here.

We define the chaoticity, $\lambda(p)$, and the weight factor, $\omega(p)$, which are usually considered to represent measures of the strength of the two- and three-pion correlations, respectively $[1,5]$ :

$$
\lambda(p)=C_{2}(p, p)-1,
$$

$$
\omega(p)=\left.\frac{C_{3}\left(p_{1}, p_{2}, p_{2}\right)-1-\left[C_{2}\left(p_{1}, p_{2}\right)-1\right]-\left[C_{2}\left(p_{2}, p_{3}\right)-1\right]-\left[C_{2}\left(p_{3}, p_{1}\right)-1\right]}{2 \sqrt{\left[C_{2}\left(p_{1}, p_{2}\right)-1\right]\left[C_{2}\left(p_{2}, p_{3}\right)-1\right]\left[C_{2}\left(p_{3}, p_{1}\right)-1\right]}}\right|_{p_{1}=p_{2}=p_{3}=p} .
$$

Note that we define the weight factor explicitly at $p_{1}=p_{2}$ $=p_{3}$, but that it has been extracted from measurements over small relative momenta [5]. The two methods show practically no difference since the weight factor is expected to be a slowly varying function of the relative momenta $[1,2]$.

The weight factor, however, is not always the measure of the strength of the genuine three-pion correlations. Generally, the three-pion correlation functions are related to the two-pion correlation functions as

$$
\begin{aligned}
C_{3}\left(p_{1}, p_{2}, p_{3}\right)= & 1+a\left[C_{2}\left(p_{1}, p_{2}\right)+C_{2}\left(p_{2}, p_{3}\right)\right. \\
& \left.+C_{2}\left(p_{3}, p_{1}\right)-3\right] \\
& +(\text { genuine three-pion correlation }) \\
& +(\text { other two-pion correlation }),
\end{aligned}
$$

where the coefficient $a$ is not always unity even at $p_{1}=p_{2}$ $=p_{3}$. Consequently, Eq. (10) does not always remove the linear dependence of $C_{2}$ 's from $C_{3}$. In the cases of a chaotic source and a partially coherent source, $a$ is unity, but for sources of more complicated structure, it is not. Furthermore, $C_{3}$ generally depends on the two-pion correlations not only as the linear $C_{2}$ 's but also in more complicated ways as a function of two momenta. We will discuss these points fully in the following section.

The relation between $C_{3}$ and $C_{2}$ depends on dynamics and is model dependent in practice. The extraction of the strength of the genuine three-pion correlations thus requires explicit knowledge of the dynamics. Accordingly, in this paper we adopt the conventional approach of using the weight factor defined as Eq. (10), for numerical results as a convenient means for making comparisons among various models.

\section{DIFFERENT MODELS OF THE SOURCE}

\section{A. Coherent source and chaotic source}

The spectra are often written by $c$-number source current $[3,6]$, yielding a chaotic source and a coherent source as the two extreme cases. The chaotic source shows the HBT effect with both the chaoticity and the weight factor as unity. The correlation functions for a coherent source are independent of the relative momenta, and the chaoticity for it is zero.

The difference between the out-state and in-state annihilation operators of an emitted pion defines the source current as 


$$
\begin{aligned}
a_{\mathrm{out}}(\mathbf{p}) & =a_{\mathrm{in}}(\mathbf{p})+i \int d^{4} x \frac{1}{\sqrt{(2 \pi)^{3} 2 p^{0}}} J(x) e^{-i p \cdot x} \\
& =a_{\mathrm{in}}(\mathbf{p})+i \frac{1}{\sqrt{(2 \pi)^{3} 2 p^{0}}} J(p),
\end{aligned}
$$

where $J(x)$ and $J(p)$ are the source current and its Fourier transform, respectively. The average that appears in Eqs. (1) -(3) can be written as

$$
\langle\cdots\rangle=\int \mathcal{D} J^{*}(p) \mathcal{D} J(p) \mathcal{P}\left[J^{*}(p), J(p)\right]\left\langle 0_{\text {in }}|\cdots| 0_{\text {in }}\right\rangle,
$$

where $\left|0_{\text {in }}\right\rangle$ is the in-state vacuum and $\mathcal{P}\left[J^{*}(p), J(p)\right]$ is the distribution functional of $J(p)$, which has a statistical fluctuation. The pion spectra are obtained in this case from Eqs. (1)-(3):

$$
\begin{gathered}
W_{1}(p)=\frac{1}{(2 \pi)^{3} 2 p^{0}}\left\langle|J(p)|^{2}\right\rangle_{J}, \\
W_{2}\left(p_{1}, p_{2}\right)=\frac{1}{(2 \pi)^{6} 2 p_{1}^{0} 2 p_{2}^{0}}\left\langle\left|J\left(p_{1}\right)\right|^{2}\left|J\left(p_{2}\right)\right|^{2}\right\rangle_{J}, \\
W_{3}\left(p_{1}, p_{2}, p_{3}\right)= \\
\quad \frac{1}{(2 \pi)^{9} 2 p_{1}^{0} 2 p_{2}^{0} 2 p_{3}^{0}} \\
\times\left\langle\left|J\left(p_{1}\right)\right|^{2}\left|J\left(p_{2}\right)\right|^{2}\left|J\left(p_{3}\right)\right|^{2}\right\rangle_{J},
\end{gathered}
$$

where $\langle\cdots\rangle_{J}=\int \mathcal{D} J^{*}(p) \mathcal{D} J(p) \mathcal{P}\left[J^{*}(p), J(p)\right] \cdots$. Hereafter, we do not explicitly show the subscript $J$ of the angle bracket. If the phase of the source current is random, we call the source chaotic. In this case, $\mathcal{P}\left[J^{*}(p), J(p)\right]$ have a Gaussian form [6] and higher-order moments of $J(p)$ are represented by the second-order moment such as

$$
\begin{aligned}
\left\langle J^{*}\left(p_{1}\right) J^{*}\left(p_{2}\right) J\left(q_{1}\right) J\left(q_{2}\right)\right\rangle= & \left\langle J^{*}\left(p_{1}\right) J\left(q_{1}\right)\right\rangle\left\langle J^{*}\left(p_{2}\right) J\left(q_{2}\right)\right\rangle \\
& +\left\langle J^{*}\left(p_{1}\right) J\left(q_{2}\right)\right\rangle \\
& \times\left\langle J^{*}\left(p_{2}\right) J\left(q_{1}\right)\right\rangle .
\end{aligned}
$$

For a chaotic source, the pion spectra are written as

$$
\begin{gathered}
W_{1}\left(p_{1}\right)=F_{11}, \\
W_{2}\left(p_{1}, p_{2}\right)=F_{11} F_{22}+F_{12}{ }^{2}, \\
W_{3}\left(p_{1}, p_{2}, p_{3}\right)=F_{11} F_{22} F_{33} \\
+\sum_{(a, b, c)} F_{a a} F_{b c}^{2}+2 F_{12} F_{23} F_{31} \\
\times \cos \left(\Phi_{12}+\Phi_{23}+\Phi_{31}\right),
\end{gathered}
$$

where the amplitude $F_{a b}$ and the phase $\Phi_{a b}$ are defined as [1]

$$
F_{a b} \exp \left(i \Phi_{a b}\right) \equiv \frac{1}{(2 \pi)^{3} \sqrt{2 p_{a}^{0} 2 p_{b}^{0}}}\left\langle J^{*}\left(p_{a}\right) J\left(p_{b}\right)\right\rangle .
$$

$\Sigma_{(a, b, c)}$ implies a sum over $(a, b, c)=(1,2,3), \quad(2,3,1)$, $(3,1,2)$. The correlation functions are then

$$
\begin{gathered}
C_{2}\left(p_{1}, p_{2}\right)=1+\frac{F_{12}^{2}}{F_{11} F_{22}}, \\
C_{3}\left(p_{1}, p_{2}, p_{3}\right)=1+\sum_{(a, b)} \frac{F_{a b}^{2}}{F_{a a} F_{b b}}+2 \frac{F_{12} F_{23} F_{31}}{F_{11} F_{22} F_{33}} \\
\times \cos \left(\Phi_{12}+\Phi_{23}+\Phi_{31}\right),
\end{gathered}
$$

where $\Sigma_{(a, b)}$ is over $(a, b)=(1,2),(2,3),(3,1) . F_{a b}$ and $\Phi_{a b}$ contain the information about the size and shape of the pionemitting source, due to the HBT effect. The chaoticity and weight factor for a chaotic source always achieve unity.

When the source current has no randomness, the source is called coherent. In this case, the $n$-pion spectra are expressed as

$$
W_{n}\left(p_{1}, \ldots, p_{n}\right)=\prod_{l=1}^{n} W_{1}\left(p_{l}\right) .
$$

The HBT effect does not appear in this case. The correlation functions achieve unity, and the chaoticity vanishes.

\section{B. Partially coherent source}

In nuclear collisions, one may expect to involve the mixture of a coherent source and a chaotic source, as suggested by the observation that the chaoticities are often smaller than unity. Here, we sketch the case of the partially coherent source, mostly following Ref. [1].

When the pion-emitting source is a mixture of a chaotic source and a coherent source, the source current is written as a sum of both currents, $J(x)=J_{\text {cha }}(x)+J_{\text {coh }}(x)$ [1,3]. The sources are not correlated with each other, $\left\langle J_{\text {cha }}^{*}(p) J_{\text {coh }}(q)\right\rangle=0$. The one-pion spectrum and correlation functions for this source are obtained as

$$
\begin{gathered}
W_{1}\left(p_{1}\right)=f_{11}+F_{11}, \\
C_{2}\left(p_{1}, p_{2}\right)=1+\frac{F_{12}^{2}+2 f_{12} F_{12} \cos \left(\Phi_{12}-\phi_{12}\right)}{\left(f_{11}+F_{11}\right)\left(f_{22}+F_{22}\right)},
\end{gathered}
$$




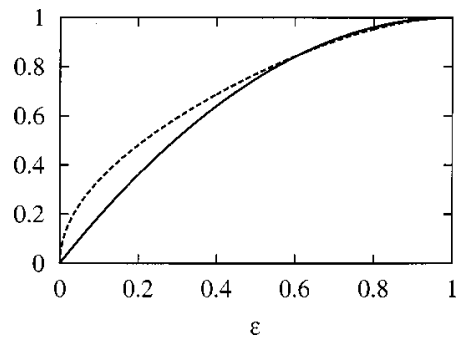

FIG. 1. Chaoticity and weight factor as functions of $\epsilon$ in the partially coherent model. The solid and dashed lines stand for the chaoticity and weight factor, respectively.

$$
\begin{aligned}
C_{3}( & \left.p_{1}, p_{2}, p_{3}\right) \\
& =1+\sum_{(a, b)} \frac{F_{a b}^{2}+2 f_{a b} F_{a b} \cos \left(\Phi_{a b}-\phi_{a b}\right)}{\left(f_{a a}+F_{a a}\right)\left(f_{b b}+F_{b b}\right)} \\
& +2 \frac{1}{\left(f_{11}+F_{11}\right)\left(f_{22}+F_{22}\right)\left(f_{33}+F_{33}\right)} \\
& \times\left\{F_{12} F_{23} F_{31} \cos \left(\Phi_{12}+\Phi_{23}+\Phi_{31}\right)\right. \\
& \left.+\sum_{(a, b, c)}\left[f_{a b} F_{b c} F_{c a} \cos \left(\phi_{a b}+\Phi_{b c}+\Phi_{c a}\right)\right]\right\},
\end{aligned}
$$

where

$$
f_{a b} \exp \left(i \phi_{a b}\right) \equiv \frac{1}{(2 \pi)^{3} \sqrt{2 p_{a}^{0} 2 p_{b}^{0}}} J_{\mathrm{coh}}^{*}\left(p_{a}\right) J_{\mathrm{coh}}\left(p_{b}\right),
$$

and $F_{a b}$ and $\Phi_{a b}$ are the same as those for a chaotic source, Eq. (21). The chaoticity and weight factor are evaluated as

$$
\begin{gathered}
\lambda(p)=\epsilon(p)[2-\epsilon(p)], \\
\omega(p)=\sqrt{\epsilon(p)} \frac{3-2 \epsilon(p)}{[2-\epsilon(p)]^{3 / 2}},
\end{gathered}
$$

where $\epsilon(p)$ is the fractional parameter of the coherent source:

$$
\epsilon\left(p_{1}\right)=\frac{F_{11}}{f_{11}+F_{11}} .
$$

The source becomes chaotic for $\epsilon(p)=1$, while it becomes coherent for $\epsilon(p)=0$. Figure 1 shows the chaoticity and weight factor as functions of $\epsilon(p)$, and in Fig. 5 the weight factor is shown as a function of chaoticity, varying as $\epsilon(p)$. The weight factor can vary from 0 to 1 . In this case, the term $-3 \lambda$, in Eq. (10), completely removes the two-pion correlations.

\section{Multicoherent sources}

We consider the mixture of a small number of coherent sources. This model differs from the case of multiple coher- ent sources that was previously examined in [3] and, to the best of our knowledge, this is the first time that this model has been explicitly discussed. We assume here that the pionemitting source is made of $N$ coherent sources that are not coherent with each other and appear to be obeying the Poisson distribution. Note that the model considered in [3] is a mixture of multiple coherent sources that are randomly distributed, and that the model was introduced as a description of a chaotic source when the number of the coherent sources becomes large. In a sense our model here is the opposite limit of the small number of coherent sources in the model considered in [3]. In the following, we present merely the final expressions, leaving their derivations for Appendix A.

The source current can be written as

$$
J(x)=\sum_{n=1}^{N} j\left(x-X_{n}\right) e^{-i \theta_{n}}
$$

where $j\left(x-X_{n}\right)$ is the $n$th coherent source current, located at $X_{n}$ with the random phase $\theta_{n}$. Each coherent source is assumed to be expressed by the same $j(x)$ but to be located at a different position. There are $N$ coherent sources, obeying the Poisson distribution. Note that the distribution must be renormalized in order to exclude the no-source event that is not observed.

The one-pion spectrum and normalized correlation functions are obtained as

$$
\begin{gathered}
W\left(p_{1}\right)=a_{1} \frac{\alpha}{1-e^{-\alpha}} \\
C_{2}\left(p_{1}, p_{2}\right)=1+\frac{\alpha}{\alpha+1}\left|\rho_{12}\right|^{2} \\
C_{3}\left(p_{1}, p_{2}, p_{3}\right)=1+\frac{\alpha(\alpha+2)}{\alpha^{2}+3 \alpha+1} \sum_{(a, b)}\left|\rho_{a b}\right|^{2} \\
+\frac{\alpha^{2}}{\alpha^{2}+3 \alpha+1} 2 \operatorname{Re}\left(\rho_{12} \rho_{23} \rho_{31}\right),
\end{gathered}
$$

where $\rho_{i j}$ is the Fourier transform of the spatial distribution of coherent sources, and $\alpha$ is a parameter of the Poisson distribution. The mean number of coherent sources is $\alpha /[1$ $-\exp (-\alpha)]$. In this case, the subtraction $-3 \lambda$, in Eq. (10),

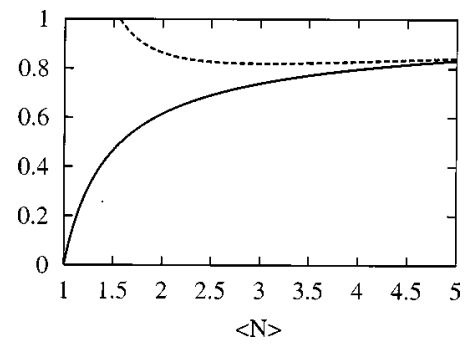

FIG. 2. Chaoticity and weight factor as functions of the mean number of coherent sources in the model of multicoherent sources. The solid and dashed lines stand for the chaoticity and weight factor, respectively. 
does not remove the two-pion correlations completely, because the coefficient of $\left|\rho_{12}\right|^{2}$ in Eq. (34) is different from that in Eq. (35). The chaoticity and weight factor are derived as

$$
\begin{gathered}
\lambda=\frac{\alpha}{\alpha+1}, \\
\omega=\frac{1}{2} \frac{2 \alpha^{2}+2 \alpha+3}{\alpha^{2}+3 \alpha+1} \sqrt{\frac{\alpha+1}{\alpha} .}
\end{gathered}
$$

In Fig. 2, the chaoticity and weight factor are illustrated as functions of the mean number of sources, and Fig. 5 shows the weight factor as a function of the chaoticity. The chaoticity varies from 0 to 1 , because this source becomes coherent at $\alpha=0$ and chaotic at $\alpha \rightarrow \infty$. The weight factor diverges at $\alpha \rightarrow 0$. The reason for this is the failure of the subtraction
$-3 \lambda$, in Eq. (10), and the divergence is not caused by the genuine three-pion correlation.

\section{Multicoherent sources and one chaotic source}

We now consider the source in which the multicoherent sources of the previous subsection are mixed with a chaotic source. The multicoherent sources are not coherent with each other, as before. The source current is written as

$$
J(x)=\sum_{n=1}^{N} j\left(x-X_{n}\right) e^{-i \theta_{n}}+J_{\text {cha }}(x),
$$

where $j(x)$ and $J_{\text {cha }}(x)$ are a coherent source current and the chaotic source current, respectively. The $n$th coherent source is located at $X_{n}$ and is distributed with $\rho\left(X_{n}\right)$. The phase, $\theta_{n}$, is randomly distributed between 0 and $2 \pi$. There are $N$ coherent sources, obeying the Poisson distribution, Eq. (A9).

The pion spectra come out to be

$$
\begin{aligned}
& W_{1}\left(p_{1}\right)=\alpha h_{11}+F_{11}, \\
& W_{2}\left(p_{1}, p_{2}\right)=W_{1}\left(p_{1}\right) W_{1}\left(p_{2}\right)+\alpha h_{11} h_{22}+\left|\alpha h_{12} e^{i \psi_{12}}+F_{12} e^{i \Phi_{12}}\right|^{2}, \\
& W_{3}\left(p_{1}, p_{2}, p_{3}\right)=W_{1}\left(p_{1}\right) W_{1}\left(p_{2}\right) W_{1}\left(p_{3}\right)+(3 \alpha+1) \alpha h_{11} h_{22} h_{33} \\
& +\alpha \sum_{(a, b, c)} h_{a a} h_{b b} F_{c c}+\sum_{(a, b, c)} W_{1}\left(p_{a}\right)\left|\alpha h_{b c} e^{i \psi_{b c}}+F_{b c} e^{i \Phi_{b c}}\right|^{2} \\
& +2 \sum_{(a, b, c)} h_{a a}\left[\alpha^{2}\left|h_{b c}\right|^{2}+\alpha h_{b c} F_{b c} \cos \left(\Phi_{b c}-\psi_{b c}\right)\right] \\
& +2 \operatorname{Re}\left\{\left(\alpha h_{12} e^{i \psi_{12}}+F_{12} e^{i \Phi_{12}}\right)\left(\alpha h_{23} e^{i \psi_{23}}+F_{23} e^{i \Phi_{23}}\right)\left(\alpha h_{31} e^{i \psi_{31}}+F_{31} e^{i \Phi_{31}}\right)\right\},
\end{aligned}
$$

where $\alpha$ is the parameter in the Poisson distribution, and

$$
h_{i j} e^{i \psi_{i j}}=\frac{j^{*}\left(p_{i}\right) j\left(p_{j}\right)}{(2 \pi)^{3} \sqrt{2 p_{1}^{0} 2 p_{2}^{0}}} \rho_{i j}
$$

For $\left|p_{1}-p_{2}\right| \rightarrow \infty$, we have

$$
\frac{W_{2}\left(p_{1}, p_{2}\right)}{W_{1}\left(p_{1}\right) W_{1}\left(p_{2}\right)} \rightarrow 1+\frac{1}{\alpha}\left[1-\epsilon\left(p_{1}\right)\right]\left[1-\epsilon\left(p_{2}\right)\right],
$$

where the fractional parameter of the chaotic source $\epsilon$ is defined as

$$
\epsilon\left(p_{1}\right)=\frac{F_{11}}{\alpha h_{11}+F_{11}} .
$$

The normalization factor of $C_{2}\left(p_{1}, p_{2}\right)$, shown in Eq. (4), does not yield the proper asymptotic value of unity as the relative momentum approaches infinity. Generally $\epsilon$ depends on the momentum, and the asymptotic value of $C_{2}$ thus depends on the two momenta separately. If we assume that $\epsilon$ is independent of the momentum, we find that $C_{2}$ is normalized properly by the use of Eqs. (4) and (5). In this case, the correlation functions are given by

$$
C_{2}\left(p_{1}, p_{2}\right)=1+\frac{\left|\alpha h_{12} e^{i \psi_{12}}+F_{12} e^{i \Phi_{12}}\right|^{2}}{W_{1}\left(p_{1}\right) W_{1}\left(p_{2}\right)\left[1+\frac{1}{\alpha}(1-\epsilon)^{2}\right]},
$$




$$
\begin{aligned}
& C_{3}\left(p_{1}, p_{2}, p_{3}\right)=1+\frac{1}{1+\frac{3 \alpha+1}{\alpha^{2}}(1-\epsilon)^{3}+\frac{3}{\alpha}(1-\epsilon)^{2} \epsilon}\left[\sum_{(a, b)} \frac{\mid \alpha h_{a b} e^{i \psi_{a b}+F_{a b} e^{\left.i \Phi_{a b}\right|^{2}}}}{W_{1}\left(p_{a}\right) W_{1}\left(p_{b}\right)}\right. \\
& +\frac{2 \operatorname{Re}\left\{\left(\alpha h_{12} e^{i \psi_{12}}+F_{12} e^{i \Phi_{12}}\right)\left(\alpha h_{23} e^{i \psi_{23}}+F_{23} e^{i \Phi_{23}}\right)\left(\alpha h_{31} e^{\left.i \psi_{31}+F_{31} e^{i \Phi_{31}}\right)}\right\}\right.}{W_{1}\left(p_{1}\right) W_{1}\left(p_{2}\right) W_{1}\left(p_{3}\right)} \\
& \left.+2 \sum_{(a, b)}\left\{\frac{\alpha(1-\epsilon) h_{a b}^{2}}{W_{1}\left(p_{a}\right) W_{1}\left(p_{b}\right)}+\frac{(1-\epsilon) h_{a b} F_{a b} \cos \left(\Phi_{a b}-\psi_{a b}\right)}{W_{1}\left(p_{a}\right) W_{1}\left(p_{b}\right)}\right\}\right] .
\end{aligned}
$$

Comparison of the preceding $C_{2}$ and the first term in the square bracket in $C_{3}$ shows that the two-pion correlations are not to be removed completely, as $a \neq 1$ in Eq. (11) in this case. The conventional weight factor thus no longer represents the strength of the genuine three-pion correlations. Furthermore, the last sum in the square bracket of the above $C_{3}$ represents the effects of the two-pion correlation (because it depends on a pair of the momenta), corresponding to the "other two-pion correlation", in Eq. (11).

The chaoticity and weight factor are obtained as

$$
\begin{gathered}
\lambda=\frac{\alpha}{\alpha+(1-\epsilon)^{2}} \\
\omega=\frac{2 \alpha^{2}+2 \alpha(1-\epsilon)^{2}+3(1-\epsilon)^{3}(1-2 \epsilon)}{2\left[\alpha^{2}+3 \alpha(1-\epsilon)^{2}+(1-\epsilon)^{3}\right]} \sqrt{\frac{\alpha+(1-\epsilon)^{2}}{\alpha}} .
\end{gathered}
$$

Figures 3 and 4 show $\lambda$ and $\omega$, respectively, as functions of $\alpha$ at $\epsilon=0.1-0.9$. In Fig. 6 , the weight factor is shown as a function of the chaoticity, varying $\epsilon$ for various $\alpha$ 's. The divergence of $\omega$ at $\alpha=0$, except for $\epsilon=0.5$, is caused by the incomplete cancellation of the $C_{2}$ 's.

\section{DISCUSSIONS AND SUMMARY}

We have examined various models of the source that are not completely chaotic. The chaoticity comes out to be between 0 and 1 in all models, but the weight factor takes a wide range of the value. The value of the weight factor even diverges in some cases, as a consequence of the incomplete removal of the two-pion correlations from $C_{3}$. For a partially

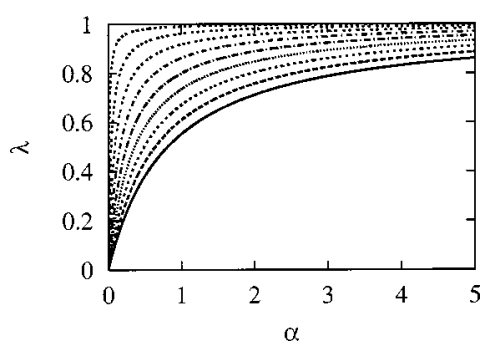

FIG. 3. Chaoticity as a function of $\alpha$ in the model of multicoherent sources and one chaotic source. The lines from down to up correspond to $\epsilon$ varying from 0.1 to 0.9 with the step 0.1 . coherent source that is commonly examined, the removal is complete and the anomalous behavior does not appear.

There is a way to avoid such incomplete removal, at least in all the models that we have examined here. It is done through a new subtraction,

$$
\begin{aligned}
R_{3}\left(p_{1}, p_{2}, p_{3}\right)= & C_{3}\left(p_{1}, p_{2}, p_{3}\right)-1 \\
& -\frac{\langle n\rangle\left(2\langle n(n-1)\rangle-\langle n\rangle^{2}\right)}{\langle n(n-1)(n-2)\rangle} \frac{\langle n(n-1)\rangle}{\langle n\rangle^{2}} \\
& \times \sum_{(a, b)}\left[C_{2}\left(p_{a}, p_{b}\right)-1\right]
\end{aligned}
$$

where $\langle n\rangle$ and similar variables are defined in Eqs. (6)-(8). This subtraction works correctly for the multicoherent sources and also for the mixture of multicoherent sources and one chaotic source (but at the zero relative momenta). We find that the terms corresponding to the "other two-pion correlations" also vanish at the zero relative momenta (see Appendix B). Using Eq. (49), we define a new weight factor as

$$
\begin{aligned}
\omega^{\prime}= & \frac{\langle n(n-1)(n-2)\rangle}{\langle n\rangle^{3}} \frac{1}{2} R_{3}\left(p_{1}, p_{2}, p_{3}\right) \\
& \times\left(\frac{\langle n(n-1)\rangle}{\langle n\rangle^{2}} \sum_{(a, b)}\left\{C_{2}\left(p_{a}, p_{b}\right)-1\right\}\right)^{-3 / 2}
\end{aligned}
$$

For the partially coherent source, the new weight factor becomes the conventional weight factor of Eq. (10).

Though the new weight factor properly represents the strength of the genuine three-pion correlations for all models

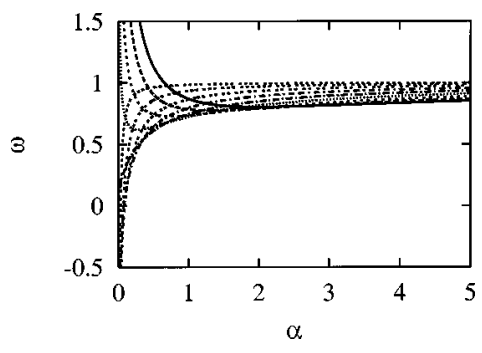

FIG. 4. Weight factor as a function of $\alpha$. The model and lines are the same as in Fig. 3. 


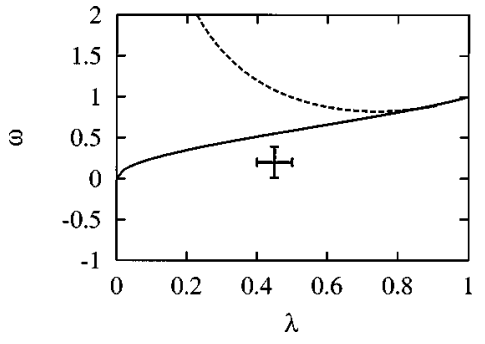

FIG. 5. Weight factors as functions of chaoticities. The solid and dashed lines stand for the partially coherent source and the multicoherent sources, respectively. The plot with error bars is the experimental data from NA44.

examined in this work, the new weight factor must not have this property at all times. The relation between $C_{2}$ 's and $C_{3}$ is generally complicated, depending on dynamics in each case. We thus do not expect that the new weight factor has a universal application.

Figures 5 and 6 illustrate the weight factors as functions of the chaoticities for the different models, together with the experimental data from the CERN NA44 Collaboration [5]. In this experiment, the chaoticity and weight factor are measured as $0.4-0.5$ and $0.20 \pm 0.19$, respectively. Figure 5 shows that the partially coherent source and the multicoherent sources disagree with the experiment. The mixture of multicoherent sources and one chaotic source reproduces the data if we set $\alpha=0.13$ and $\epsilon=0.60$. This corresponds to the mean number of the coherent sources being 0.13 and to about $60 \%$ of the total pions emitted from the chaotic source. The ratio of the pion number emitted from one coherent source to that from one chaotic source, $h_{11} / F_{11}$, is then about 5 . This ratio may be unrealistically large, but we note that the experimental data are "minimum bias," suggesting that our best fit may not be unrealistic since the multiplicity fluctuation can be large. Further data are needed to confirm that this is indeed the case.

In summary, we investigate the two- and three-pion correlations for various models of a source that is not completely chaotic. The chaoticity and weight factor are evaluated as measures of two- and three-pion correlations. The chaoticity always varies between 0 and 1 , but the weight factor takes the value of a wide range and sometimes even diverges. The conventional weight factor includes the effects

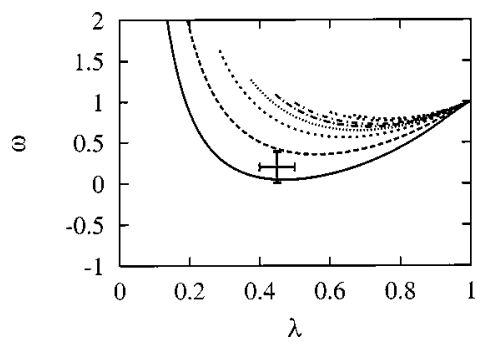

FIG. 6. Weight factors as functions of chaoticities in the model of multicoherent sources and one chaotic source, varying $\epsilon$ from 0 to 1 . The lines from down to up stand for $\alpha=0.1,0.2,0.4,0.6,0.8$, $1.0,1.5,2.0$, respectively. The plot with error bars is the experimental data from NA44. of the two-pion correlations in some models, yielding the anomalous behavior. We propose, in all models considered here, a new weight factor that has no such difficulty, but not expected to be valid universally. We find that the model of multicoherent sources and one chaotic source could reproduce the chaoticity and the weight factor observed in the recent experiment.

\section{ACKNOWLEDGMENTS}

This work was supported by the U.S. Department of Energy under Grant No. DE-FG03-87ER40347 at SCUN, and by the National Science Foundation under Grant Nos. PHY9420470 and PHY-9412818 at Caltech.

\section{APPENDIX A: MULTICOHERENT SOURCE MODEL}

We assume that $N$ coherent sources are created during a collision and that the position of the $n$th source is $X_{n}$. The sources are uncorrelated with each other. A source current is defined as

$$
J(x)=\sum_{n=1}^{N} j\left(x-X_{n}\right) e^{-i \theta_{n}}
$$

where $\theta_{n}$ is a unique random number varying from 0 to $2 \pi$. The average about $\theta_{n}$ is denoted by $\langle\cdots\rangle$. The Fourier transform of the source current is written as

$$
J(p)=\sum_{n=1}^{N} j(p) e^{i p \cdot X_{n}-i \theta_{n}}
$$

where $j(p)$ is the Fourier transform of $j(x)$. The one-pion spectrum is

$$
\begin{aligned}
W_{N}\left(p_{1}\right) & =\frac{1}{(2 \pi)^{3} p_{1}^{0}}\left\langle\left|J\left(p_{1}\right)\right|^{2}\right\rangle \\
& =\frac{\left|j\left(p_{1}\right)\right|^{2}}{(2 \pi)^{3} p_{1}^{0}} \sum_{n, m}^{N} e^{-i p_{1} \cdot\left(X_{n}-X_{m}\right)}\left\langle e^{i \theta_{n}-i \theta_{m}}\right\rangle \\
& =a_{1} N
\end{aligned}
$$

where $p^{0}$ is on-shell and

$$
a_{1}=\frac{\left|j\left(p_{1}\right)\right|^{2}}{(2 \pi)^{3} p_{1}^{0}} .
$$

The two-pion spectrum is

$$
\begin{aligned}
W_{N}\left(p_{1}, p_{2}\right) & =\frac{1}{(2 \pi)^{6} p_{1}^{0} p_{2}^{0}}\left\langle\left|J\left(p_{1}\right)\right|^{2}\left|J\left(p_{2}\right)\right|^{2}\right\rangle \\
& =a_{1} a_{2}\left[N^{2}+\sum_{n \neq m}^{N} e^{-i\left(p_{1}-p_{2}\right) \cdot\left(X_{m}-X_{n}\right)}\right] .
\end{aligned}
$$


The location of each small source $X_{n}$ is assumed to obey a distribution $\rho\left(X_{n}\right)$, which is normalized to be unity, or $\int \rho\left(X_{n}\right) d^{4} X_{n}=1$. Averaging the two-pion spectrum with the distribution, we obtain

where $\rho_{12}=\int \rho(x) e^{-i\left(p_{1}-p_{2}\right) \cdot x} d^{4} X$. Note that $\Sigma_{i, j} A_{i j}$ $=\sum_{i} A_{i i}+\sum_{i \neq j} A_{i j}$ and $\sum_{i \neq j} 1=N(N-1)$.

The three-pion spectrum is

$W_{N}\left(p_{1}, p_{2}, p_{3}\right)=\frac{1}{(2 \pi)^{6} p_{1}^{0} p_{2}^{0} p_{3}^{0}}\left\langle\left|J\left(p_{1}\right)\right|^{2}\left|J\left(p_{2}\right)\right|^{2}\left|J\left(p_{3}\right)\right|^{2}\right\rangle$,

and the averaged spectrum is evaluated as

$$
\begin{aligned}
\bar{W}_{N}\left(p_{1}, p_{2}, p_{3}\right)= & a_{1} a_{2} a_{3} \\
& \times\left[N^{3}+N^{2}(N-1)\left(\left|\rho_{12}\right|^{2}+\left|\rho_{23}\right|^{2}+\left|\rho_{31}\right|^{2}\right)\right. \\
& \left.+N(N-1)(N-2) 2 \operatorname{Re}\left(\rho_{12} \rho_{23} \rho_{31}\right)\right] .
\end{aligned}
$$

Note that $\quad \sum_{i, j, k} A_{i j k}=\sum_{i} A_{i i i}+\sum_{i \neq j}\left(A_{i i j}+A_{i j i}+A_{j i i}\right)$ $+\sum_{i \neq j \neq k} A_{i j k}$ and that $\Sigma_{i \neq j \neq k} 1=N(N-1)(N-2)$.

We next assume that the coherent sources obey the Poisson distribution. The Poisson distribution is

$$
P_{N}^{(\text {usual })}=\frac{\alpha^{N}}{N !} e^{-\alpha} \quad \text { for } \quad N=0 \sim \infty .
$$

The $N=0$ event should be excluded because in such an event no pions are emitted. The distribution we use is renormalized as

$$
P_{N}=\frac{\alpha^{N}}{N !} \frac{1}{e^{\alpha}-1} \quad \text { for } \quad N=1 \sim \infty .
$$

The expectation values change from those of the usual Poisson distribution,

$$
\langle N\rangle_{P}=\frac{\alpha}{1-e^{-\alpha}},
$$

$$
\begin{aligned}
\bar{W}_{N}\left(p_{1}, p_{2}\right) & =\int \rho\left(X_{1}\right) d X_{1} \cdots \rho\left(X_{N}\right) d^{4} X_{N} W_{N}\left(p_{1}, p_{2}\right) \\
& =a_{1} a_{2}\left[N^{2}+N(N-1)\left|\rho_{12}\right|^{2}\right],
\end{aligned}
$$

$$
\begin{gathered}
\langle N(N-1)\rangle_{P}=\frac{\alpha^{2}}{1-e^{-\alpha}}, \\
\langle N(N-1) \cdots(N-n)\rangle_{P}=\frac{\alpha^{n+1}}{1-e^{-\alpha}} .
\end{gathered}
$$

Pion spectra averaged with the Poisson distribution, $W$ $=\sum_{N=1}^{\infty} P_{N} \bar{W}_{N}$, are

$$
W\left(p_{1}\right)=a_{1} \frac{\alpha}{1-e^{-\alpha}},
$$

$$
W\left(p_{1}, p_{2}\right)=\frac{a_{1} a_{2}}{1-e^{-\alpha}}\left[\alpha(\alpha+1)+\alpha^{2}\left|\rho_{12}\right|^{2}\right],
$$

$$
\begin{aligned}
W\left(p_{1}, p_{2}, p_{3}\right)= & \frac{a_{1} a_{2} a_{3}}{1-e^{-\alpha}}\left[\alpha\left(\alpha^{2}+3 \alpha+1\right)\right. \\
& +\alpha^{2}(\alpha+2)\left(\left|\rho_{12}\right|^{2}+\left|\rho_{23}\right|^{2}+\left|\rho_{31}\right|^{2}\right) \\
& \left.+\alpha^{3} 2 \operatorname{Re}\left(\rho_{12} \rho_{23} \rho_{31}\right)\right] .
\end{aligned}
$$

The correlation functions are

$$
C_{2}\left(p_{1}, p_{2}\right)=1+\frac{\alpha}{\alpha+1}\left|\rho_{12}\right|^{2}
$$

$$
\begin{aligned}
C_{3}\left(p_{1}, p_{2}, p_{3}\right)= & 1+\frac{\alpha(\alpha+2)}{\alpha^{2}+3 \alpha+1}\left(\left|\rho_{12}\right|^{2}+\left|\rho_{23}\right|^{2}+\left|\rho_{31}\right|^{2}\right) \\
& +\frac{\alpha^{2}}{\alpha^{2}+3 \alpha+1} 2 \operatorname{Re}\left(\rho_{12} \rho_{23} \rho_{31}\right),
\end{aligned}
$$

where the correlation functions are normalized to be unity at $\left|p_{i}-p_{j}\right| \rightarrow \infty$. The chaoticity and weight factor are

$$
\lambda=\frac{\alpha}{\alpha+1},
$$

$$
\omega=\frac{1}{2} \frac{2 \alpha^{2}+2 \alpha+3}{\alpha^{2}+3 \alpha+1} \sqrt{\frac{\alpha+1}{\alpha}} .
$$

APPENDIX B: DERIVATION OF EQ. (50)

The two- and three-pion spectra can be written generally as

$$
\begin{gathered}
W_{2}\left(p_{1}, p_{2}\right)=\left\langle a_{1}^{\dagger} a_{1}\right\rangle\left\langle a_{2}^{\dagger} a_{2}\right\rangle+\left\langle a_{1}^{\dagger} a_{2}\right\rangle\left\langle a_{2}^{\dagger} a_{1}\right\rangle+\left\langle\left\langle a_{1}^{\dagger} a_{2}^{\dagger} a_{1} a_{2}\right\rangle\right\rangle, \\
W_{3}\left(p_{1}, p_{2}, p_{3}\right)=\left\langle a_{1}^{\dagger} a_{1}\right\rangle\left\langle a_{2}^{\dagger} a_{2}\right\rangle\left\langle a_{3}^{\dagger} a_{3}\right\rangle+\sum_{(a, b, c)}\left\langle a_{a}^{\dagger} a_{a}\right\rangle\left\langle a_{b}^{\dagger} a_{c}\right\rangle\left\langle a_{c}^{\dagger} a_{b}\right\rangle+2 \operatorname{Re}\left(\left\langle a_{1}^{\dagger} a_{2}\right\rangle\left\langle a_{2}^{\dagger} a_{3}\right\rangle\left\langle a_{3}^{\dagger} a_{1}\right\rangle\right) \\
+\sum_{(a, b, c)}\left\langle a_{a}^{\dagger} a_{a}\right\rangle\left\langle\left\langle a_{b}^{\dagger} a_{c}^{\dagger} a_{b} a_{c}\right\rangle\right\rangle+2 \operatorname{Re} \sum_{(a, b, c)}\left\langle a_{a}^{\dagger} a_{b}\right\rangle\left\langle\left\langle a_{b}^{\dagger} a_{c}^{\dagger} a_{a} a_{c}\right\rangle\right\rangle+\left\langle\left\langle a_{1}^{\dagger} a_{2}^{\dagger} a_{3}^{\dagger} a_{1} a_{2} a_{3}\right\rangle\right\rangle,
\end{gathered}
$$


where $a_{i}$ is an annihilation operator of momentum $p_{i}$. We assume that $\left\langle a_{i}\right\rangle=0$, and therefore we do not consider the coherent source and partially coherent source in this appendix. $\langle\langle\cdots\rangle\rangle$ corresponds to a cumulant, or a connected Green function. If we have a generating functional, $Z\left[z^{*}(p), z(p)\right]$, such as

$$
\left\langle a_{1}^{\dagger} \cdots a_{n}^{\dagger} a_{1} \cdots a_{n}\right\rangle=\left.\frac{\delta^{2 n} Z\left[z^{*}(p), z(p)\right]}{\delta z\left(p_{1}\right) \cdots \delta z\left(p_{n}\right) \delta z^{*}\left(p_{1}\right) \cdots \delta z\left(p_{n}\right)}\right|_{z=0},
$$

then the cumulant is obtained as

$$
\left\langle\left\langle a_{1}^{\dagger} \cdots a_{n}^{\dagger} a_{1} \cdots a_{n}\right\rangle\right\rangle=\left.\frac{\delta^{2 n}\left(\ln Z\left[z^{*}(p), z(p)\right]\right)}{\delta z\left(p_{1}\right) \cdots \delta z\left(p_{n}\right) \delta z^{*}\left(p_{1}\right) \cdots \delta z\left(p_{n}\right)}\right|_{z=0} .
$$

We introduce two assumptions. The first is

$$
\begin{gathered}
\left\langle\left\langle a_{1}^{\dagger} a_{2}^{\dagger} a_{1} a_{2}\right\rangle\right\rangle=A\left\langle a_{1}^{\dagger} a_{1}\right\rangle\left\langle a_{2}^{\dagger} a_{2}\right\rangle, \\
\left\langle\left\langle a_{1}^{\dagger} a_{2}^{\dagger} a_{3}^{\dagger} a_{1} a_{2} a_{3}\right\rangle\right\rangle=B\left\langle a_{1}^{\dagger} a_{1}\right\rangle\left\langle a_{2}^{\dagger} a_{2}\right\rangle\left\langle a_{3}^{\dagger} a_{3}\right\rangle,
\end{gathered}
$$

where $A$ and $B$ are constant. The second assumption is that the interference terms, such as $\left\langle a_{1}^{\dagger} a_{2}\right\rangle\left\langle a_{2}^{\dagger} a_{1}\right\rangle$, vanish by integrating over the momenta, for example,

$$
\int d^{3} p_{1} d^{3} p_{2}\left\langle a_{1}^{\dagger} a_{2}\right\rangle\left\langle a_{2}^{\dagger} a_{1}\right\rangle=0
$$

Thus we can obtain

$$
\begin{gathered}
\langle n(n-1)\rangle=(1+A)\langle n\rangle^{2}, \\
\langle n(n-1)(n-2)\rangle=(1+3 A+B)\langle n\rangle^{3} .
\end{gathered}
$$

Correlation functions at the zero relative momenta become

$$
\begin{gathered}
C_{2}(p, p)=1+\frac{1}{1+A}, \\
C_{3}(p, p, p)=1+\frac{1}{1+3 A+B}\{3+2+6 A\} .
\end{gathered}
$$

The terms 3, 2, and $6 A$ between the braces in the above equation correspond to $\sum_{(a, b, c)}\left\langle a_{a}^{\dagger} a_{a}\right\rangle\left\langle a_{b}^{\dagger} a_{c}\right\rangle\left\langle a_{c}^{\dagger} a_{b}\right\rangle$, $2 \operatorname{Re}\left(\left\langle a_{1}^{\dagger} a_{2}\right\rangle\left\langle a_{2}^{\dagger} a_{3}\right\rangle\left\langle a_{3}^{\dagger} a_{1}\right\rangle\right)$, and $2 \operatorname{Re} \sum_{(a, b, c)}\left\langle a_{a}^{\dagger} a_{b}\right\rangle\left\langle\left\langle a_{b}^{\dagger} a_{c}^{\dagger} a_{a} a_{c}\right\rangle\right\rangle$ in Eq. (B2). To obtain the genuine three-pion correlation, we should subtract the first and third terms between the braces. Thus we define the new weight factor, considering the above discussion, as

$$
\begin{aligned}
\omega^{\prime} & =\frac{1}{2} \frac{(1+3 A+B)\left[C_{3}(p, p, p)-1\right]-3(1+2 A)\left[C_{2}(p, p)-1\right]}{\left[(1+A)\left(C_{2}(p, p)-1\right)\right]^{3 / 2}} \\
& =\frac{1}{2} \frac{\frac{\langle n(n-1)(n-2)\rangle}{\langle n\rangle^{3}}\left[C_{3}(p, p, p)-1\right]-3 \frac{2\langle n(n-1)\rangle-\langle n\rangle^{2}}{\langle n\rangle^{2}}\left[C_{2}(p, p)-1\right]}{\left[\frac{\langle n(n-1)\rangle}{\langle n\rangle^{2}}\left(C_{2}(p, p)-1\right)\right]^{3 / 2}} .
\end{aligned}
$$

This equation corresponds to Eq. (50).

The two assumptions are valid for multicoherent sources and one chaotic source with setting $A=\alpha^{-1}(1-\epsilon)^{2}$ and $B$ $=\alpha^{-2}(1-\epsilon)^{3}$. For multicoherent sources, the assumptions are not valid because of the factor $[1-\exp (-\alpha)]^{-1}$, in Eqs. (A14)-(A16), due to the renormalized Poisson distribution, Eq. (A10). In using the usual Poisson distribution instead of the renormalized one, the factor vanishes, and the assumptions become applicable with $A=\alpha^{-1}$ and $B=\alpha^{-2}$. The correlation functions are the same both in the cases of the usual and renormalized distributions. If we use the renormalized distributions and other assumptions, we can derive Eq. (B12), but the derivation is more complicated. 
[1] U. Heinz and Q. H. Zhang, Phys. Rev. C 56, 426 (1997).

[2] H. Nakamura and R. Seki (unpublished).

[3] M. Gyulassy, S. K. Kauffmann, and L. W. Wilson, Phys. Rev. C 20, 2267 (1979).

[4] M. Biyajima, A. Bartl, T. Mizoguchi, O. Terazawa, and N.
Suzuki, Prog. Theor. Phys. 84, 931 (1990).

[5] NA44 Collaboration, H. Bøggild et al., Phys. Lett. B 455, 77 (1999).

[6] I. V. Andreev, M. Plümer, and R. M. Weiner, Int. J. Mod. Phys. A 8, 4577 (1993). 